\title{
Evaluación de la Dispersión de Polen Como Medida de Bioseguridad para la Liberación de Plantas Transgénicas de Papa a Campo
}

\author{
W. Bravo ${ }^{1}$, Javier Franco ${ }^{2}$, Gladys Main ${ }^{2}$, \\ Enrique Carrasco ${ }^{2}$, Julio Gabriel ${ }^{2}$
}

\begin{abstract}
Resumen
Con el objetivo de evaluar el riesgo de diseminación de polen de cultivares de papa (4x) y fortalecer las normas de bioseguridad en relación a la liberación de plantas transgénicas, en la Estación Experimental Toralapa a $3430 \mathrm{msnm}$ (Cochabamba,-Bolivia) se estudió la dispersión de polen del cultivo de papa utilizando las variedades Waych'a (4x) y Revolución (androestéril 4x) siguiendo el Diseño Sistemático Circular (DSC) con 20 tratamientos y 4 repeticiones. Se estableció la distancia de desplazamiento de polen, y se identificaron los factores responsables de dicha dispersión. El estudio se complementó con el monitoreo de especies silvestres presentes en parcelas cultivadas y no cultivadas próximas al campo experimental y su potencial de cruzabilidad con especies cultivadas. Se constató que la dispersión alcanzó los $20 \mathrm{~m}$ de distancia desde la parcela fuente, pero el mayor porcentaje de plantas polinizadas se encontraron a menores distancias (1-5 m) que a mayores distancias (18-20 m). La dispersión fue influida tanto por el viento como por insectos, siendo Bombus spp. el insecto con mayor frecuencia de aproximación a las flores. Se identificó a la especie Solanum toralapanum $(2 x)$ como la especie silvestre más frecuente en parcelas cultivadas próximas al ensayo, pero que bajo condiciones de invernadero no fue posible su hibridación con el cultivar nativo Waych'a.
\end{abstract}

Palabras claves adicionales: $\quad$ androestéril, bioseguridad, polinización, hibridación, OGM.

1 Parte de tesis para obtener el título de Ingeniero Agrónomo, UMSS, Cbba, Bolivia.

2 Fundación PROINPA (Promoción e Investigación de Productos Andinos), Casilla 4285, Cbba, Bolivia. 


\title{
Evaluation of Pollen Dispersal as a Biosafety Measure for the Release of Transgenic Potato Plants in Fields
}

\begin{abstract}
Summary
To evaluate the potential risk of potato pollen dissemination and to strengthen biosafety regulation in relation to the use of transgenic potato plants, in Toralapa Experimental station of 3430 masl. (Cochabamba-Bolivia), the pollen dispersion was studied by using the potato cultivars Waych'a (4x) and Revolution (malesterile $4 \mathrm{x}$ ) in a Systematic Circular Design with 20 treatments and 4 replications. The distance of pollen dissemination reached up to $20 \mathrm{~m}$ from the centre plot (pollen donor) but the highest number of pollinated plants were found within shorter distances (1-5m) than further away (18-20m). The pollen dissemination was dependant on both, the wind (intensity and direction) and insects, being Bombus sp. the most frequent one. The wild potato species Solanum toralapanum $(2 x)$ was the most common in nearby cultivated potato fields but under greenhouse conditions this was not able to cross with the cultivated cv. Waych'a (4x).
\end{abstract}

Additional índex words:

malesterile, biosafety, hybridization, polinization, GMO.

\section{Introducción}

La papa Solanum spp. es el cultivo más importante de la zona andina de muchos países de América Latina y constituye uno de los cuatro cultivos más importantes en el ámbito mundial. Los fitomejoradores hace algunos años están generando por medio de técnicas biotecnológicas Organismos Genéticamente Modificados (OGM), que incluyen las plantas transgénicas, es decir, plantas que tienen dentro de su genoma genes foráneos de cualquier organismo o sintético incorporado por técnicas de laboratorio que no involucran cruzamientos. Dada la rápida evolución y expansión de está tecnología, es por lo tanto necesario desarrollar medidas de bioseguridad que contribuyan a preservar el ecosistema y la biodiversidad debido a los posibles riesgos ambientales (7). Se teme que las nuevas características o genes incorporados podrían transformar la planta en maleza, afectar la germinación, la tolerancia a sequías, heladas y otros efectos no deseados (4). Los cultivos transgénicos podrían cruzarse con variedades nativas o especies silvestres y dar lugar a descendencia híbrida con mayor potencial 
para convertirse en maleza, desplazando a las poblaciones pequeñas de su hábitat natural que no podrían competir con estos híbridos y seguir generando nuevos híbridos transgénicos por el continuo intercambio de polen que ocurre en la naturaleza. Bolivia constituye uno de los centros de origen y diversidad de la papa Solanum spp. (8). El flujo génico entre especies cultivables y parientes silvestres tiene lugar como resultado de una propagación cruzada natural mediante la transferencia de genes por medio del polen de las flores $(2,6)$. Estos antecedentes hacen necesario efectuar estudios para evaluar el riesgo que representaría el desplazamiento y las distancias de dispersión del polen en campo e identificar los factores responsables de esta dispersión en el área de estudio, con el fin de diseñar medidas apropiadas de bioseguridad, para evitar dicha dispersión. Siendo la variedad más importante en las zonas andinas de mayor producción de papa en Bolivia, este estudio se dedicará al cultivar Waych'a (Solanum tuberosum subsp. andígena, 4x). Así mismo es importante determinar el tipo de especies silvestres presentes, su localización y finalmente su cruzabilidad con papas cultivadas como el progenitor Waych'a.

\section{Materiales y métodos}

El presente estudio se realizó en la Estación Experimental Toralapa de la Fundación PROINPA, Cochabamba, Bolivia, durante la campaña Agrícola 97/98. Se usaron como material vegetal la variedad Waych'a (Solanum tuberosum ssp andigena, 4x), cultivar comercial de abundante floración que fue utilizado como progenitor masculino donador de polen y la variedad Revolución (híbrido S.t. ssp. tuberosum x S.t. ssp. andigena, 4x) como receptor de polen, cuya característica particular es ser macho estéril (androestéril). Para estudiar la distancia de diseminación de polen en campo se empleó el Diseño Sistemático Circular (DSC), en el cual se asignaron dos parcelas distintas: en la primera se sembró la variedad Waych'a (Parcela Central) y en la segunda se sembró la variedad Revolución, donde se ubicaron los distintos tratamientos. El experimento consistió en 20 tratamientos y 4 repeticiones. Cada tratamiento estaba representado por una población de plantas (surco) en forma de círculos, distribuidos sistemática y correlativamente (T1, T2, T3, T20; el T1 a un metro; el T2 a dos metros, etc.). Se usó cada 14 de círculo como repetición: la repetición I orientada hacia NO, la repetición II al SO, la repetición III al SE y finalmente la repetición IV al NE (Figura 1). La densidad de siembra fue $0.30 \times 1.0 \mathrm{~m}$. Durante el ensayo se realizaron las labores culturales de deshierbe, aporque, control fitosanitario, etc. Se efectuó dos riegos, pero en general se dependió de la precipitación pluvial que alcanzó un promedió de 378 mm, muy inferior con relación al promedio normal de $691 \mathrm{~mm}$.

Se evaluó como variable de respuesta el número de plantas que formaron frutos en los diferentes tratamientos y repeticiones. Las semillas extraídas de los frutos cosechados fueron tratados con ácido giberélico (AG3) y sembrados en almácigos para evaluar su viabilidad y comparar las 
características florales de la progenie de Revolución x Waych'a utilizando descriptores morfológicos (5)

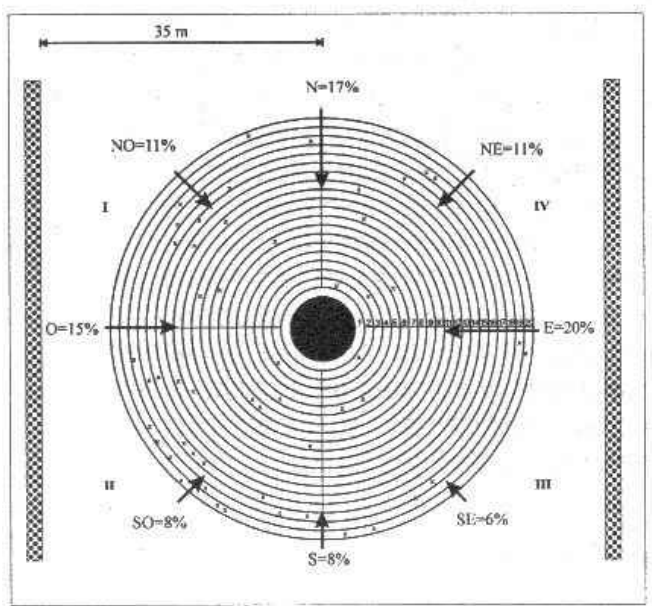

\begin{tabular}{|l} 
REFERENCIAS \\
Waych'a \\
Revolución \\
Barrera Vegetal (Cebada) \\
Dirección del viento (\%) \\
I, II, III, IV Repeticiones (Cuadrantes) \\
$1,2, \ldots, 20 \quad$ Tratamientos (Surcos \\
Plantas con bayas
\end{tabular}

Figura 1. Campo experimental mostrando la distribución) de las variedades de papa, los 20 tratamientos (surcos) en las cuatro repeticiones (I:NO; II:SO; III:SE; IV:NE), la barrera vegetal, dirección de viento predominante y la presencia de plantas con bayas.

Para el análisis estadístico se utilizó la prueba de $\mathrm{x} 2$ para ambas variables de respuesta. También se estimaron las correlaciones posibles entre el porcentaje de plantas con frutos y las distintas distancias.

Además durante el experimento se estudiaron los factores ambientales (bióticos y abióticos) que podían estar involucrados en la dispersión y diseminación del polen. Para el efecto se instaló una veleta en el mismo ensayo, y se registraron tres lecturas diarias (08:00 a.m., 12:00m. y 18:00 p.m.) tanto para la dirección como para la velocidad del viento durante el periodo de floración. Durante el período de floración (seis semanas de duración) se registraron 60 horas de observación de los insectos que presentaban proximidad a las flores de papa para el estudio de la polinización entomófila y se realizaron capturas de estos para su respectiva identificación. Los muestreos de los insectos se efectuaron tres veces por semana durante dos a tres horas por día.

El otro estudio importante para complementar las medidas de bioseguridad, fue el de identificar la presencia de especies silvestres, y con este propósito se realizaron monitoreos de estás especies en parcelas cultivadas y no 
cultivadas, registrándose la distancia a la que se encontraban con respecto a la parcela de ensayo. Para determinar el potencial de hibridación de las especies silvestres encontradas, se realizaron cruzas dirigidas entre S.t. ssp. andigena $\times$ S. acaule y S. toralapanum, estos últimas fueron emasculadas antes de la polinización artificial en condiciones de invernadero.

\section{Resultados y discusión}

Polinización: La formación de frutos localizados a diferentes distancias de la parcela central revela el cruzamiento natural entre los cultivares Revolución $x$ Waych'a y que el polen del cultivo de papa puede Tabla 1. Prueba de X2 para la variable de número de plantas de papa con bayas del cV. Revolución.

\begin{tabular}{lccc}
\hline \multicolumn{1}{c}{ F.V } & G.L & $\begin{array}{c}\text { Chi } \\
\text { cuadrado }\end{array}$ & P $>$ Chi \\
\hline Bloques & 3 & 2.0080 & 0.1112 \\
Tratamientos & 19 & 38.1942 & $0.0056^{\star}$ \\
\hline
\end{tabular}

*Diferencia significativa a P:0.05 dispersarse desde la parcela fuente hasta los 20 metros de distancia (Figura 1). La prueba de x2 mostró diferencias significativas a nivel del $0.05 \%$ en el número de plantas que formaron bayas en las distintas distancias o tratamientos (Tabla 1) Las comparaciones ortogonales entre las medias de los tratamientos (Tabla 2) mostraron diferencias significativas entre: T1-T11, T1-T15, T5-T11, T5-T15 y T5-T20, indicando diferencias entre los primeros tratamientos y los tratamientos intermedios y extremos, mas no entre los intermedios y extremos.

Tabla2.

Comparaciones ortogonales entre las medias de los tratamientos para el número de plantas con bayas.

\begin{tabular}{lcc}
\hline Contrastes & Chi cuadrado & P>Chi \\
\hline T1 vs. T5 & 0.0002 NS & 0.9896 \\
T1 vs. T11 & $7.9054^{\star}$ & 0.0049 \\
T1 vs. T15 & $7.6282^{\star}$ & 0.0057 \\
T5 vs. T11 & $9.4374^{\star}$ & 0.0021 \\
T5 vs. T15 & $9.1237^{\star}$ & 0.0025 \\
T5 vs. T20 & $7.9035^{\star}$ & 0.0049 \\
T11 vs. T15 & 0.7398 NS & 0.3897 \\
T1 1 vs. T20 & 2.0857 NS & 0.1487 \\
T15vs. T20 & 0.3318 NS & 0.5646 \\
\hline
\end{tabular}

*:Significativo a P 0.05; NS: No significativo; T: tratamientos 
Por otro lado, de acuerdo a los análisis de regresión realizados, con el objeto de estudiar el efecto de los factores ambientales (bióticos y abióticos) sobre el comportamiento de la población y determinar si la presencia de plantas con bayas por la dispersión de polen estaba asociada con las distancias predispuestas en el ensayo, se evidenció respuestas diferentes (Figura 2). Se observó en primer lugar, una regresión lineal significativa de dependencia del número de plantas polinizadas por efecto de las distancias en las repeticiones III (SE) y IV (NE), no así en las repeticiones I (NO) y II (SO). Es decir, en las primeras dos repeticiones indicadas, el porcentaje de plantas con frutos fue mayor a distancias cortas $(\mathrm{T} 1, \mathrm{~T} 5, \mathrm{~T} 8)$ y a medida que se incrementaban las distancias, el porcentaje de plantas también fue disminuyendo. La mayor formación de bayas en los primeros surcos y con porcentajes mayores que el resto, indicaría que el factor eólico influyó en la polinización de estas plantas. Por otra parte, se aprecia que en las repeticiones I y II, la polinización de plantas ocurrió a partir de los 5 y 8 metros de distancia, y la ausencia de plantas polinizadas en los primeros surcos 0 tratamientos podría atribuirse a variaciones en la intensidad y dirección (E-O: 20\%) del viento. En la misma figura (Figura 2), se muestran las correlaciones calculadas para determinar el grado de asociación entre el número de plantas con bayas en relación a las distancias de la dispersión de polen en los tratamientos para cada una de las repeticiones del ensayo. Los mayores valores de correlación correspondieron a las repeticiones III $\left(r^{2}=77 \%\right)$ y IV $\left(r^{2}=88 \%\right)$ y las menores correlaciones a las repeticiones I $\left(r^{2}=40 \%\right)$ y II $\left(r^{2}=56 \%\right)$.

Las correlaciones altas de las repeticiones III y IV se deberían a la influencia del viento que polinizó las plantas de los primeros surcos (T1, T5), porque probablemente la concentración de polen en el aire fue mayor en las plantas más próximas a la parcela central con Waych'a y a medida que el polen se alejaba de la parcela central, su permanencia en el aire fue disminuyendo. En cambio, en la repetición I y II, con valores de correlación bajos o de pobre asociación entre las distancias de dispersión de polen y plantas que formaron frutos, el factor biótico (insectos) sería el que más influyó en la polinización, ya que se localizó mayor porcentaje de plantas polinizadas a mayores distancias, es decir a los bordes de la repetición II (T18, T19, T20) y no así en los primeros tratamientos (T1, T5 y T8). Este resultado concuerda con otros estudios dónde se evidenció mayor movimiento y permanencia de Bombus spp. en los bordes de la parcela (9). Por otro lado, los análisis basados en descriptores morfológicos de las plantas originadas de las bayas del cv. Revolución en campo (progenies), indican que la formación de bayas se debió principalmente a la polinización cruzada de ambos progenitores y que la formación de híbridos confirma la transferencia de genes entre los dos progenitores. 


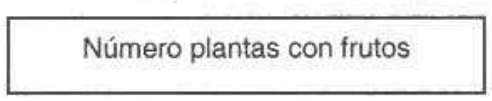

Repetición I (NO)

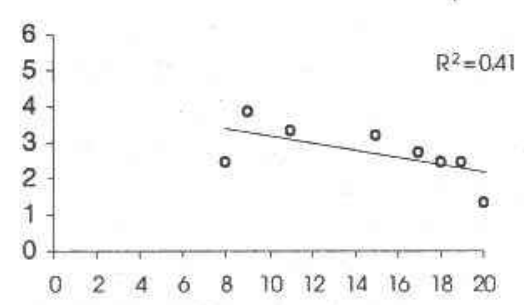

Repetición II (SO)

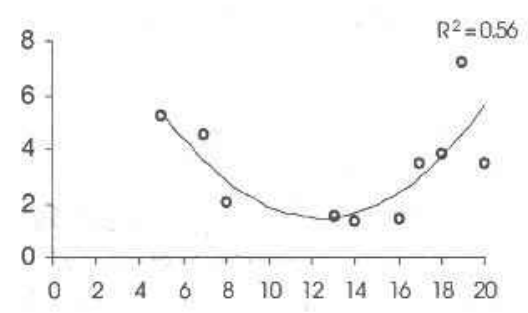

\section{Distancia de dispersión (m)}

Repetición IV (NE)

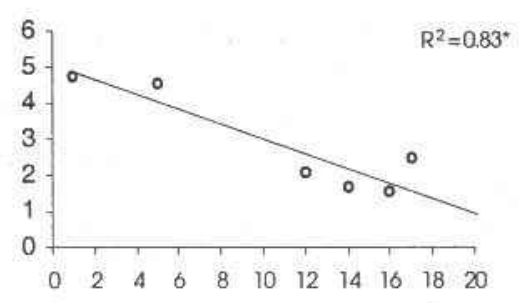

Repetición III (SE)

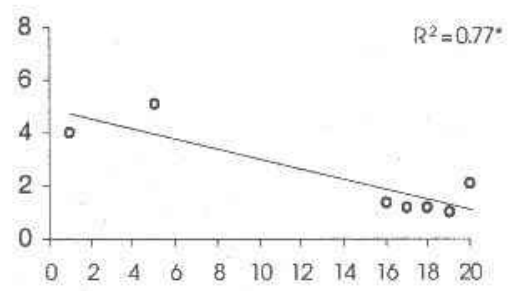

Figura 2. Análisis de regresión y correlación de la variable número de plantas con frutos en relación con distancias de dispersión de polen en las cuatro repeticiones.

Especies Silvestres: Por el monitoreo efectuado en inmediaciones del ensayo se identificó a la especie silvestre de papa S. toralapanum en gran proporción, la misma que se encontraba en parcelas cultivadas y no cultivadas de papa. Las distancias a las que las plantas de S. toralapanum fueron ubicadas oscilaron entre 50 a 500 metros del ensayo. Otra de las especies identificadas fue $\mathrm{S}$. acaule que se encontró fuera del radio de acción del ensayo, es decir, en otra zona papera ubicada a más de 1 km de distancia.

Cruzabilidad: Los cruzamientos dirigidos entre Revolución x Waych'a en invernadero fueron 24 que equivalieron a un total de 106 flores polinizadas y cuya eficiencia llegó al $86 \%$ a través de la formación de bayas. Esta eficiencia de polinización fue superior a la polinización libre que ocurrió en 
campo, en la que el viento y/o insectos tuvieron una influencia limitada o poco eficiente.

Estos resultados de eficiencia en la polinización (libre y dirigida) por lo tanto indican que a medida que los factores bióticos-abióticos se expresen de la mejor manera en un determinado ambiente, éstos determinarán la eficiencia de polinización. Si la intervención de los factores ambientales (viento/insecto) fuera considerable ó favorable, cualquier variedad incluyendo a las modificadas genéticamente de papa, fuera potencialmente eficiente para polinizar a otras especies parentales si en su camino no enfrentaran alguna barrera física ó genética. Este último concepto se relaciona al fracaso en obtener progenies de los cruzamientos entre $S$. toralapanum $(2 x) \times$ Waych'a $(4 x)$. La inhabilidad de producir híbridos se debe a dos causas, en primer lugar al desbalance cromosómico entre Waych'a (4x) y S. toralapanum (2x) que crea infertilidad génica (3). En segundo lugar, la otra causa fue el desbalance de los factores embrión: endosperma (3:4) que ocasionó el balance inviable que no permitió, la formación de híbridos (1), ya que una relación normal que crea balance viable es de 2:3. En las cruzas con $S$. acaule, si bien tanto Waych'a como $S$. acaule tienen número de cromosomas idéntico (4x), no se reportó cruzamiento exitoso alguno. Estos resultados también pueden ser explicados desde el punto de vista del NBE (Número de Balance del Endospermo), dónde la relación entre éstas dio 5:3, lo que explica por qué estas dos especies no tuvieron éxito a pesar de tener igual número de ploidia. Los cruzamientos entre Revolución x Waycha dieron una relación $2: 3$, es decir, una doble fertilización normal similar al cruzamiento entre diploides, lo que produjo un balance viable y, por consiguiente, la formación de la semilla. Sin embargo, los cruzamientos de especies cultivables, (4x) $x$ especies silvestres $(2 \mathrm{x})$ puede tener lugar vía gametos $2 \mathrm{n}$. En nuestro estudio se observaron granos de polen de $\mathrm{S}$. toralapanum con un diámetro de 12(i, lo que indica que no es una especie (2n) y por lo tanto, por esta vía, tampoco fue posible el cruzamiento con la papa cultivada Waych'a.

Por lo indicado anteriormente, se tiene que bajo condiciones naturales de campo y de altura, y por la formación de híbridos entre los cruzamientos Revolución x Waych'a, la dispersión de polen por viento e insectos (Bombus spp.) alcanzó los $20 \mathrm{~m}$ y esta dispersión fue mayor en los primeros surcos, disminuyendo a mayores distancias, registrándose así una asociación entre dispersión de polen y distancia. La diseminación de polen fue influida por factores ambientales del lugar y por Bombus spp. (Familia Bombinae), que fue la especie vector más frecuente en la polinización en relación a otros insectos polinizadores como abejas y mariposas. Así mismo, se detectó la presencia de las especies silvestres $S$. acaule y $S$. toralapanum en las parcelas cultivadas, presentándose esta última con mayor frecuencia, pero sin la posibilidad de que ocurra una hibridación con las papas cultivadas aun bajo condiciones de invernadero. Estos resultados indican que al ser posible la diseminación o polinización libre hasta los $20 \mathrm{~m}$ de distancia como consecuencia de los factores abióticos y bióticos en condiciones de Toralapa, es importante ampliar este 
tipo de estudios y considerar antes de la liberación de plantas transgénicas, tanto el factor genético de las especies involucradas (cultivadas y silvestres) en la zona de liberación, como el empleo de elementos de contención o barreras físicas que impidan la diseminación de polen, reduciendo el riesgo potencial de contaminación de genes a otras plantas. De lo contrario, las plantas transformadas deberían también ser modificadas genéticamente por androesterilidad, eliminándose así la ocurrencia de cruzamientos naturales no deseados.

\section{Referencias Bibliográficas}

1. Centro Internacional de la Papa. 1984. Manual de Manejo de Germoplasma de Papa, Documento de Capacitación. Departamento de Capacitación y Comunicación. Lima, Perú. 38 pp.

2. Dale, P. J. 1992. Spread of engineered genes to wild relatives. Plant Physiology 100: 13-15.

3. Den Ninjs, T.P.M., S.J. Pelouquin. 1977. $2 n$ gametes in potato species and their function in sexual polyploidization. Euphytica 26 : 586-600.

4. Frederick, R.J., I. Virgin, E. Lindarte. 1995. Riesgos ambientales de las plantas transgénicas en centros de diversidad: la papa como un modelo. Memorias del Taller Regional el 2 y 3 de junio de 1995 en el Parque Nacional Iguazú, Argentina. Comisión Consultiva Internacional sobre Biotecnología- Instituto Interamericano de Cooperación para la Agricultura. Caracas, Venezuela. 89 pp.

5. Lujan, L. 1997. Morfología, estructura y fisiología de la planta de papa. FEDEPAPA, Bogotá, Colombia: 23-29.

6. Mac Partlan, H.C., P.J. Dale. 1994. An assessment of gene transfer by pollen from field grown transgenic potatoes to non -transgenic potatoes and wild species. Transgenic Research 3: 216-225.

7. Mac Neely, J.A. 1994. Critical issues in the implementation of the convention on Biological Diversity. In: A.F. Krattiger et al. (Eds.) Widening Perspectives on Biodiversity. pp. 7-10. IUCN, Switzerland and International Academy of the Environmental, Geneva, Switzerland.

8. Ochoa, C.M. 1990. The potatoes of South America: Bolivia. Cambridge University Press, Cambridge, England. 111 pp.

9. Van Marrewijk. 1994. Flowering biology and hybrid varieties: Flowering and pollination. International Course on Applied Plant Breeding. International Agriculture Center, Wageningen, The Netherlands. $45 p$ 